\title{
Structure of completely positive quantum master equations with memory kernel
}

\author{
Heinz-Peter Breuer ${ }^{1, *}$ and Bassano Vacchini ${ }^{2, \oplus}$ \\ ${ }^{1}$ Physikalisches Institut, Universität Freiburg, Hermann-Herder-Strasse 3, D-79104 Freiburg, Germany \\ ${ }^{2}$ Dipartimento di Fisica dell'Università di Milano and INFN Sezione di Milano, Via Celoria 16, I-20133 Milano, Italy
}

(Dated: November 13, 2018)

\begin{abstract}
Semi-Markov processes represent a well known and widely used class of random processes in classical probability theory. Here, we develop an extension of this type of non-Markovian dynamics to the quantum regime. This extension is demonstrated to yield quantum master equations with memory kernels which allow the formulation of explicit conditions for the complete positivity of the corresponding quantum dynamical maps, thus leading to important insights into the structural characterization of the non-Markovian quantum dynamics of open systems. Explicit examples are analyzed in detail.
\end{abstract}

PACS numbers: 03.65.Yz,42.50.Lc,02.50.Ga,03.65.Ta

\section{INTRODUCTION}

Dissipation, damping and dephasing phenomena in the dynamics of open quantum systems can often be modelled through the standard techniques of the theory of quantum Markov processes in which the open system's density matrix is governed by a quantum master equation with Lindblad structure [1, 2]. However, in the description of complex quantum mechanical systems one encounters in many physically relevant cases a complicated non-Markovian behavior [3] that cannot be described by means of these standard methods. In fact, non-Markovian systems feature strong memory effects, finite revival times caused by long-range correlation functions, and non-exponential damping and decoherence which generally render impossible a theoretical treatment through a dynamical semigroup (see, e.g., Refs. [4, 5, 6, 7, 8, 9, 10, 11, 12, 13]). As a consequence the analysis of non-Markovian quantum dynamics is extremely demanding. Even in the regime of classical probability theory it is difficult to formulate general equations of motion for the probability distributions of non-Markovian processes. In quantum mechanics the situation is even more involved since the classical condition of the preservation of the positivity for the distribution functions is to be replaced by the stronger condition of complete positivity of the resulting quantum dynamical maps.

In classical probability theory and the theory of stochastic processes there exists however a well established and widely used class of non-Markovian processes, namely the class of semi-Markov processes [14, 15, 16, $17,18]$. It is thus natural to investigate possible generalizations of this type of processes to the quantum case. Recently we have proposed such a generalization, leading to the concept of a quantum semi-Markov process [19]. In the present paper we elaborate the details of

\footnotetext{
*Electronic address: breuer@physik.uni-freiburg.de
}

${ }^{\dagger}$ Electronic address: vacchini@mi.infn.it this approach and indicate a number of further examples of applications of the theory. The class of quantum processes constructed here is demonstrated to yield generalized master equations with memory kernel, and to allow the mathematical formulation of necessary and even necessary and sufficient conditions which ensure the complete positivity of the corresponding quantum dynamical maps. Indeed the formulation of such conditions for nonMarkovian master equations is a highly nontrivial task [4, 20, 21, 22].

The paper is organized as follows. Section II contains a short introduction into the theory of classical semiMarkov processes. We define the fundamental quantities, such as the semi-Markov matrix, the survival probabilities and the waiting time distributions, derive the generalized master equation and the structure of the classical memory kernel, and discuss several examples for classical memory functions and waiting time distributions. The generalization of these concepts to the quantum case is developed in Sec. III] We introduce a class of quantum master equations with memory kernel and formulate explicitly the corresponding conditions for the complete positivity of the quantum time evolution. A number of examples and applications is also discussed. Finally, some conclusions are drawn in Sec. IV.

\section{CLASSICAL SEMI-MARKOV PROCESSES}

In the present section we want to give a brief introduction to classical semi-Markov processes, focusing on the basic quantities necessary in order to describe and uniquely determine such processes. In particular building on these quantities we will be able to explicitly derive a generalized master equation for the time evolution of the conditional transition probabilities of the process, which is the starting point for a generalization to the quantum case. General references to the subject are typically found in the mathematics literature [14, 15, 16] (see also the monograph [17]), even though examples of classical semi-Markov processes have been extensively studied in the physics literature under the name continuous time 
random walk (see [18] for a comprehensive treatment and references therein).

\section{A. Semi-Markov matrix}

Semi-Markov processes naturally generalize Markov processes by combining the theory of Markov chains and of renewal processes [23]. In a Markov chain a system jumps among different states according to certain probabilities depending on departure and arrival state, the time spent in a given state being immaterial. A renewal process is instead a counting process in which the times among successive events are independent identically distributed random variables characterized by an arbitrary common waiting time distribution, the adjective renewal stressing the fact that the process starts anew at every step. If this waiting time distribution is of exponential type one obtains as a special case of renewal process a Poisson process, in fact the exponential is the only memoryless distribution leading to a Markov counting process. In this case knowing that a system has already been in a state for a given amount of time provides no additional information on the expected time of the next jump. By combining the two features a semi-Markov process describes a system moving among different states according to fixed transition probabilities, so that the sequence of visited states forms a Markov chain, spending a random time in each state. These random sojourn times however are described by a waiting time distribution which is not necessarily of exponential type, as in a Markov process, and which might depend both on the present state and on the immediately following one. If one only considers the different states visited by a semi-Markov process one recovers a Markov chain, while if the state space is reduced to a single point one recovers a renewal process.

A semi-Markov process is uniquely determined introducing a so-called semi-Markov matrix $Q_{m n}(\tau)$, which gives the probabilities for a jump from a state $n$ to a state $m$ in a time $\tau$. More precisely, given that the process arrived in the state $n$ at time $t, Q_{m n}(\tau)$ denotes the probability that it jumps to the next state $m$ no later than time $t+\tau$. The semi-Markov matrix can be expressed through the corresponding densities $q_{m n}(\tau)$ defined by

$$
d Q_{m n}(\tau)=q_{m n}(\tau) d \tau
$$

which represent a collection of state dependent waiting time distributions. If a jump eventually occurs with certainty the following normalization holds

$$
\sum_{m} \int_{0}^{+\infty} d \tau q_{m n}(\tau)=1 .
$$

In terms of the state dependent waiting time distribution $q_{m n}(\tau)$ one can naturally introduce the survival probability

$$
g_{n}(\tau)=1-\sum_{m} \int_{0}^{\tau} d s q_{m n}(s)
$$

that is the probability not to have left state $n$ by time $\tau$. For the special case in which the waiting time distribution for the next jump to take place does only depend on the initial state one has the factorization

$$
q_{m n}(\tau)=\pi_{m n} f_{n}(\tau)
$$

with $\pi_{m n}$ the transition probabilities of the corresponding Markov chain satisfying $\sum_{m} \pi_{m n}=1$, and $f_{n}(\tau)$ a normalized waiting time distribution. Correspondingly one also has the factorization $Q_{m n}(\tau)=\pi_{m n} F_{n}(\tau)$, with $F_{n}(\tau)$ the cumulative distribution function providing the probability of a jump out of state $n$ in a time $\tau$. If the system can get stuck in some state $n$, the corresponding $f_{n}(\tau)$ is not normalized to one and (2) becomes a strict inequality.

It is of interest to consider the form of $q_{m n}(\tau)$ corresponding to a Markov process. Such a process is recovered for a factorizing expression of the form

$$
q_{m n}(\tau)=\pi_{m n} \lambda_{n} e^{-\lambda_{n} \tau},
$$

with corresponding survival probability given by

$$
g_{n}(\tau)=e^{-\lambda_{n} \tau} .
$$

Denoting by $\hat{h}(u)$ the Laplace transform of a function $h(\tau)$ defined on the positive real line,

$$
\hat{h}(u)=\int_{0}^{+\infty} d \tau h(\tau) e^{-u \tau},
$$

we observe for later use that in Laplace representation semi-Markov matrix and survival probability for a Markov process read

$$
\hat{q}_{m n}(u)=\pi_{m n} \frac{\lambda_{n}}{u+\lambda_{n}}
$$

and

$$
\hat{g}_{n}=\frac{1}{u+\lambda_{n}},
$$

respectively. This choice corresponds to an exponential waiting time distribution $f_{n}(\tau)=\lambda_{n} e^{-\lambda_{n} \tau}$, leading to the following memoryless property. Let us denote by $\tau_{n}$ the random variable giving the time spent in state $n$, and let us consider the conditional probability for a jump out of $n$ to take place after a time $t+s$, given that no jump has taken place up to time $s$, one immediately has from ([6)

$$
P\left\{\tau_{n}>t+s \mid \tau_{n}>s\right\}=\frac{P\left\{\tau_{n}>t+s\right\}}{P\left\{\tau_{n}>s\right\}}=e^{-\lambda_{n} t},
$$

so that this conditional probability does not depend on the time already spent in site $n$. This lack of memory only holds for an exponential distribution, whose survival probability is given by the simple exponential (6). For all other possible choices of the semi-Markov matrix semiMarkov processes are indeed non-Markovian. 


\section{B. Generalized master equation}

We now want to obtain a generalized master equation for the conditional transition probabilities of a classical semi-Markov process starting from the central quantity given by the semi-Markov matrix $q_{m n}(\tau)$. Such a generalized master equation is the counterpart for the nonMarkovian case of the Pauli master equation, which is reobtained as a special case when memory effects are absent. To do this we will follow a straightforward and intuitive path, exploiting an analog of the Kolmogorov forward equation for standard Markov processes, written in Laplace representation. Another slightly more indirect route can be found in 24], which already represented an endeavour to give a simple derivation of the generalized master equation. The point is not entirely trivial, as can be seen from the amount of literature devoted in the physics community to relate continuous time random walks, which provide examples of semi-Markov processes, to generalized master equations (see, e. g., [25] and references therein).

As a starting point we consider the Kolmogorov forward equations for a Markov process, which can be immediately written down using arguments of probabilistic nature [15]. We denote by $T_{m n}(t)$ the conditional transition probability, i. e. the probability for the process to be in the state $m$ at time $t$ under the condition that it started in state $n$ at time zero. These quantities obey the equation

$T_{m n}(t)=\delta_{m n} e^{-\lambda_{n} t}+\int_{0}^{t} d \tau \sum_{k} e^{-\lambda_{m}(t-\tau)} \pi_{m k} \lambda_{k} T_{k n}(\tau)$

the two terms on the r.h.s corresponding to contributions in which the system has performed zero or at least one jump respectively. Thus the first expression on the r.h.s. gives the probability not to have left state $n$, expressed by means of the survival probability of a Markov process $g_{n}(\tau)=e^{-\lambda_{n} \tau}$. The second expression argues on the last jump performed, summing over paths in which the system goes from state $n$ to a state $k$ in a time $\tau$ and makes at this point his last jump from $k$ to $m$, with probability density $\pi_{m k} \lambda_{k}$, dwelling there for the remaining time $t-\tau$. This equation is most easily dealt with in Laplace representation, coming to

$$
\hat{T}_{m n}(u)=\delta_{m n} \hat{g}_{m}(u)+\sum_{k} \hat{g}_{m}(u) \pi_{m k} \lambda_{k} \hat{T}_{k n}(u),
$$

and further recalling (8) and (9)

$$
\hat{T}_{m n}(u)=\delta_{m n} \hat{g}_{m}(u)+\sum_{k} \hat{g}_{m}(u) \frac{\hat{q}_{m k}(u)}{\hat{g}_{k}(u)} \hat{T}_{k n}(u)
$$

where the ratio between Laplace transform of semiMarkov matrix and survival probability appears, which we will generally denote as

$$
\hat{W}_{m k}(u)=\frac{\hat{q}_{m k}(u)}{\hat{g}_{k}(u)} .
$$

We have thus recast the Kolmogorov forward equations in a form where only the semi-Markov matrix and the related survival probability appear, starting from their specific expressions for the case of a Markov process. We now extend these equations to allow for a general semi-Markov matrix, thus obtaining a set of equations playing the role of Kolmogorov forward equations for a semi-Markov process, first obtained by Feller [14]. Recalling that due to Eq. (3) the general expression for the Laplace transform of the survival probability in terms of the semi-Markov matrix is given by

$$
\hat{g}_{n}(u)=\frac{1-\sum_{m} \hat{q}_{m n}(u)}{u},
$$

and subtracting the term $\sum_{k} \hat{q}_{k m}(u) \hat{T}_{m n}(u)$ from both sides of Eq. (13) one comes to

$$
\begin{aligned}
& \hat{T}_{m n}(u)\left[1-\sum_{k} \hat{q}_{k m}(u)\right]=\delta_{m n} \hat{g}_{m}(u) \\
& +\hat{g}_{m}(u) \sum_{k} \frac{\hat{q}_{m k}(u)}{\hat{g}_{k}(u)} \hat{T}_{k n}(u)-\sum_{k} \hat{q}_{k m}(u) \hat{T}_{m n}(u),
\end{aligned}
$$

and finally diving by $\hat{g}_{m}(u)$ one obtains

$$
u \hat{T}_{m n}(u)-\delta_{m n}=\sum_{k}\left[\hat{W}_{m k}(u) \hat{T}_{k n}(u)-\hat{W}_{k m}(u) \hat{T}_{m n}(u)\right] .
$$

Taking the inverse Laplace transformation of this equation and using $T_{m n}(0)=\delta_{m n}$ one is thus immediately led to the generalized master equation

$$
\begin{aligned}
\frac{d}{d t} T_{m n}(t)=\int_{0}^{t} d \tau \sum_{k}[ & W_{m k}(\tau) T_{k n}(t-\tau) \\
& \left.-W_{k m}(\tau) T_{m n}(t-\tau)\right] .
\end{aligned}
$$

Denoting by $P_{n}(t)$ the probability to be in state $n$ at time $t$ starting from a fixed state at the initial time zero one can also write this equation as

$$
\begin{aligned}
\frac{d}{d t} P_{n}(t)=\int_{0}^{t} d \tau \sum_{m}\left[W_{n m}(\tau) P_{m}(t-\tau)\right. & \\
& \left.-W_{m n}(\tau) P_{n}(t-\tau)\right] .
\end{aligned}
$$

\section{Classical Memory kernel}

The matrix of functions $W_{n m}(t)$ can be naturally called classical memory kernel, and is given by the inverse Laplace transform of Eq. (14), expressed in the time domain through

$$
q_{m n}(\tau)=\int_{0}^{\tau} d s W_{m n}(s) g_{n}(\tau-s) \equiv\left(W_{m n} * g_{n}\right)(\tau),
$$


where $*$ denotes as usual the convolution product, or more compactly in terms of the Laplace transformed quantities

$$
\hat{W}_{m n}(u)=\frac{\hat{q}_{m n}(u)}{\hat{g}_{n}(u)}=\frac{u \hat{q}_{m n}(u)}{1-\sum_{l} \hat{q}_{l n}(u)} .
$$

As one immediately checks, in the Markovian case the memory kernel is given by a matrix of positive constants times a delta function

$$
W_{m n}(t)=\Gamma_{m n} 2 \delta(t)
$$

with

$$
\Gamma_{m n}=\pi_{m n} \lambda_{n}
$$

thus satisfying

$$
\Gamma_{m n} \geq 0, \quad \sum_{m} \Gamma_{m n}=\lambda_{n},
$$

leading to the usual Pauli master equation

$$
\frac{d}{d t} P_{n}(t)=\sum_{m}\left[\Gamma_{n m} P_{m}(t)-\Gamma_{m n} P_{n}(t)\right] .
$$

Note in particular that the positivity and the normalization of the coefficients $\Gamma_{n m}$ naturally allow an interpretation as transition probabilities per unit time, i. e., as transition rates, a simple picture which is no more available in the general case. In fact, the functions $W_{n m}(t)$ can take on negative values even when obtained from a well-defined semi-Markov matrix, as we will show with simple examples.

To do this let us first consider in detail the situation described in Eq. (4), corresponding to factorized contributions in the semi-Markov matrix [24]. We note that in this case the survival probability simply reads

$$
g_{n}(\tau)=1-\int_{0}^{\tau} d s f_{n}(s)
$$

implying for the memory kernel a factorized expression of the form

$$
W_{m n}(t)=\pi_{m n} k_{n}(t)
$$

where the memory functions $k_{m}(t)$ relate waiting time distribution $f_{n}(\tau)$ and survival probability $g_{n}(\tau)$ through the integral relation

$$
f_{n}(\tau)=\int_{0}^{\tau} d s k_{n}(s) g_{n}(\tau-s)=\left(k_{n} * g_{n}\right)(\tau)
$$

corresponding to Eq. (20). Also in this case it is convenient to express these identities in the Laplace domain, so that one has

$$
\hat{g}_{n}(u)=\frac{1-\hat{f}_{n}(u)}{u}
$$

leading to a factorized expression for the memory kernel

$$
\hat{W}_{m n}(u)=\pi_{m n} \hat{k}_{n}(u)=\pi_{m n} \frac{\hat{f}_{n}(u)}{\hat{g}_{n}(u)},
$$

which together with Eq. (29) yields the following one-toone correspondence between $\hat{k}_{n}(u)$ and $\hat{f}_{n}(u)$,

$$
\hat{k}_{n}(u)=\frac{u \hat{f}_{n}(u)}{1-\hat{f}_{n}(u)} \text {. }
$$

This relation provides the most direct way to obtain the memory function $k_{n}(t)$ given a certain waiting time distribution $f_{n}(t)$.

It immediately appears from Eq. (27) that the positivity of the matrix elements of the memory kernel for the considered class of factorized expressions depends on the positivity of the memory functions $k_{n}(t)$. We will now consider simple and natural examples of waiting time distributions leading to negative memory functions, at variance with what happens in the Markovian case. To this end the dependence on the index $n$ is not relevant, since we are only interested in showing that a well-defined waiting time distribution $f(\tau)$ associated to a fixed state of the system can correspond to a negative function $k(t)$. This point will turn out to be of particular relevance in the quantum extension of the model, both in order to identify the class of admissible memory kernels together with possible pitfalls, and to make contact with relevant physical models.

Let us consider a general class of waiting time distributions given by the so-called special Erlang distributions (of order $a \in \mathbb{N}$ )

$$
f^{(a)}(\tau)=\lambda \frac{(\lambda \tau)^{a-1}}{(a-1) !} e^{-\lambda \tau},
$$

whose Laplace transform is simply given by

$$
\hat{f}^{(a)}(u)=\left(\frac{\lambda}{u+\lambda}\right)^{a} .
$$

Such a distribution describes a random variable given by the sum of $a$ independent identically distributed exponential random variables with the same positive parameter $\lambda$. Exploiting the relation (31) and inverting the Laplace transform, which is easily done since we are dealing with rational functions, one obtains for the first three orders

$$
\begin{aligned}
f^{(1)}(\tau) & =\lambda e^{-\lambda \tau} & k^{(1)}(t) & =2 \lambda \delta(t) \\
f^{(2)}(\tau) & =\lambda^{2} \tau e^{-\lambda \tau} & k^{(2)}(t) & =\lambda^{2} e^{-2 \lambda t} \\
f^{(3)}(\tau) & =\frac{\lambda^{3}}{2} \tau^{2} e^{-\lambda \tau} & k^{(3)}(t) & =\frac{2 \lambda^{2}}{\sqrt{3}} \sin (\sqrt{3 \lambda} t / 2) e^{-3 \lambda t / 2}
\end{aligned}
$$

so that for $a=3$ one indeed has negative contributions in the memory kernel. On similar grounds one can consider 
a sum of exponential random variables characterized by different parameters, still obtaining a rational function for the Laplace transform of the waiting time distributions, corresponding to so-called generalized Erlang distributions. Their expression is given by

$$
f_{(a)}(\tau)=\sum_{i}^{a}\left(\prod_{j \neq i} \frac{\lambda_{j}}{\lambda_{j}-\lambda_{i}}\right) \lambda_{i} e^{-\lambda_{i} \tau},
$$

and correspondingly

$$
\hat{f}_{(a)}(u)=\prod_{i}^{a} \frac{\lambda_{i}}{u+\lambda_{i}}
$$

In this case for $a=1$ one is obviously back to a simple exponential distribution, while for $a=2$ the waiting time distribution is a difference of two exponential functions

$$
f_{(2)}(\tau)=\frac{\lambda_{1} \lambda_{2}}{\lambda_{2}-\lambda_{1}}\left(e^{-\lambda_{1} \tau}-e^{-\lambda_{2} \tau}\right)
$$

leading to the following positive memory function

$$
k_{(2)}(t)=\lambda_{1} \lambda_{2} e^{-\left(\lambda_{1}+\lambda_{2}\right) t} .
$$

For $a=3$ depending on the value of the three parameters $\left\{\lambda_{i}\right\}_{i=1 \ldots 3}$ the memory function can become oscillatory, thus taking on negative values, according to

$$
k_{(3)}(t)=\lambda_{1} \lambda_{2} \lambda_{3} \frac{e^{\lambda_{+} t}-e^{\lambda_{-} t}}{\lambda_{+}-\lambda_{-}},
$$

with

$$
\lambda_{ \pm}=-\frac{\lambda_{1}+\lambda_{2}+\lambda_{3}}{2} \pm \frac{1}{2} \sqrt{\left(\lambda_{1}-\lambda_{2}-\lambda_{3}\right)^{2}-4 \lambda_{2} \lambda_{3}} .
$$

A complementary example is obtained taking rather than a sum of exponential random variables a single random variable with a waiting time distribution given by a multi-exponential, that is to say a convex mixture of exponential distributions

$$
f(\tau)=\sum_{i} p_{i} \lambda_{i} e^{-\lambda_{i} \tau}, \quad p_{i} \geq 0, \quad \sum_{i} p_{i}=1 .
$$

Already for the simplest nontrivial case given by a biexponential distribution

$$
f(\tau)=p \lambda_{1} e^{-\lambda_{1} \tau}+(1-p) \lambda_{2} e^{-\lambda_{2} \tau}
$$

with $0<p<1$, one obtains a memory function taking on negative values according to

$$
k(t)=\langle\lambda\rangle\left[2 \delta(t)-\frac{\Delta \lambda^{2}}{\langle\lambda\rangle} e^{-\left(p \lambda_{2}+(1-p) \lambda_{1}\right) t}\right],
$$

where with obvious notation $\langle\lambda\rangle=p \lambda_{1}+(1-p) \lambda_{2}$ and $\Delta \lambda^{2}=\left\langle\lambda^{2}\right\rangle-\langle\lambda\rangle^{2}$. It is important to stress that the function given by Eq. (37) cannot be interpreted as a multi-exponential distribution, since the weights in the sum over $i$ are not always positive. Indeed the situations described by Erlang or multi-exponential distributions correspond to two complementary pictures. In both cases the system moves from one state to another in various unobserved stages or steps, each taking an exponentially distributed time. In the case of an Erlang distribution such as Eqs. (32) or (37) however the different steps are taken in series, while for a multi-exponential distribution as given by Eq. (43) the different stages are entered in parallel, following one of the available possibilities, each with its own weight. In both cases one describes a nonMarkovian situation in terms of elementary Markovian building blocks described by exponential distributions, the non-Markovian features appearing because one does not have information on the fictitious intermediate steps. For a suitable choice of weights and parameters one can approximate any distribution by combination of stages in series and in parallel, so that these examples are in fact quite representative.

\section{QUANTUM SEMI-MARKOV PROCESSES}

\section{A. Quantum Markov processes}

In the Markovian regime the dynamics of the density matrix $\rho(t)$ of an open quantum system is governed by a master equation of the relatively simple form of a firstorder differential equation,

$$
\frac{d}{d t} \rho(t)=\mathcal{L} \rho(t)
$$

where $\mathcal{L}$ is a time-independent infinitesimal generator with the general structure

$$
\mathcal{L} \rho=-i[H, \rho]+\sum_{\alpha} \gamma_{\alpha}\left[A_{\alpha} \rho A_{\alpha}^{\dagger}-\frac{1}{2}\left\{A_{\alpha}^{\dagger} A_{\alpha}, \rho\right\}\right]
$$

The Hamiltonian $H$ describes the coherent part of the time evolution, while the $A_{\alpha}$ are operators representing the various decay modes, with $\gamma_{\alpha} \geq 0$ the corresponding positive decay rates. The solution of Eq. (46) can be written in terms of a linear map $V(t)=\exp (\mathcal{L} t)$ that transforms the initial state $\rho(0)$ into the state $\rho(t)$ at time $t \geq 0$,

$$
\rho(0) \longrightarrow \rho(t)=V(t) \rho(0) .
$$

The map $V(t)$ is a well-defined quantum dynamical map provided it preserves trace and positivity when applied to a general initial state $\rho(0)$. This is granted if $V(t)$ is a completely positive map, in accordance with general physical principles [3]. This property implies that it can be written in the Kraus form

$$
V(t) \rho(0)=\sum_{\alpha} \Omega_{\alpha}(t) \rho(0) \Omega_{\alpha}^{\dagger}(t)
$$


where in order to grant preservation of the trace the operators $\Omega_{\alpha}(t)$ have the property that the sum $\sum_{\alpha} \Omega_{\alpha}^{\dagger}(t) \Omega_{\alpha}(t)$ is equal to the unit operator. Hence, $V(t)$ represents a completely positive dynamical semigroup known as quantum Markov process. The master equation (46) leads to such a semigroup if and only if the generator is of the form of Eq. (47). This is the content of the celebrated Gorini-Kossakowski-Sudarshan-Lindblad theorem [1, 2], of paramount importance in both fundamental and phenomenological approaches to the description of irreversible dynamics in quantum mechanics [3].

For the case in which one has a closed system of equations for the populations $P_{n}(t)=\langle n|\rho(t)| n\rangle$ in a fixed orthonormal basis one recovers from Eq. (46) and Eq.(47) the Pauli master equation (25). This justifies the notion of a quantum Markov process and provides a direct connection to a classical Markov process.

\section{B. Master equations with memory kernel}

A natural non-Markovian generalization of Eq. (46) is given by the integrodifferential equation

$$
\frac{d}{d t} \rho(t)=\int_{0}^{t} d \tau \mathcal{K}(\tau) \rho(t-\tau) .
$$

Here quantum memory effects are taken into account through the introduction of the memory kernel $\mathcal{K}(\tau)$, which means that the rate of change of the state $\rho(t)$ at time $t$ depends on the states $\rho(t-\tau)$ at previous times $t-\tau$. Equations of the form (50) typically arise in the standard Nakajima-Zwanzig projection operator technique [26, 27]. As an important limiting case, the Markovian master equation (46) is recovered for a memory kernel proportional to a $\delta$-function,

$$
\mathcal{K}(\tau)=2 \delta(\tau) \mathcal{L} .
$$

To be physically acceptable the superoperator $\mathcal{K}(\tau)$ appearing in Eq. (50) must lead to a completely positive quantum dynamical map $V(t)$. The general structural characterization of the memory kernels with this property is an unsolved problem of central importance in the field of non-Markovian quantum dynamics [4, 22, 28, 29]. In fact, even the most simple and natural choices for $\mathcal{K}(\tau)$ can lead to unphysical results [4, 20]. Here we will construct a class of memory kernels which arises naturally as a quantum mechanical generalization of the classical semi-Markov processes, and allows the formulation of criteria that guarantee complete positivity.

We consider memory kernels with the general structure

$$
\begin{aligned}
\mathcal{K}(\tau) \rho= & -i[H(\tau), \rho]-\frac{1}{2} \sum_{\alpha} \gamma_{\alpha}(\tau)\left\{A_{\alpha}^{\dagger}(\tau) A_{\alpha}(\tau), \rho\right\} \\
& +\sum_{\alpha} \gamma_{\alpha}(\tau) A_{\alpha}(\tau) \rho A_{\alpha}^{\dagger}(\tau)
\end{aligned}
$$

that is to say of the form given by Eq. (47) apart from the time dependence of the operators $A_{\alpha}(\tau)$ and of the real functions $\gamma_{\alpha}(\tau)$. As previously done in the Markovian case let us consider the situation in which the populations obey a closed system of equations of motion, which then takes the form (19) of the generalized master equation for a classical semi-Markov process, where the memory kernel is given by

$$
W_{n m}(\tau)=\sum_{\alpha} \gamma_{\alpha}(\tau)\left|\left\langle n\left|A_{\alpha}(\tau)\right| m\right\rangle\right|^{2} .
$$

Thus, whenever the populations obey closed equations, Eq. (46) yields the classical Markovian master equation (25), while Eq. (50) with the kernel (52) leads under the same conditions to the generalized master equation (19) for a classical semi-Markov process. This justifies the name quantum semi-Markov process.

\section{Conditions for complete positivity}

Our next goal is the formulation of sufficient conditions that guarantee the complete positivity of the dynamical map $V(t)$ corresponding to the non-Markovian master equation defined by Eqs. (50) and (52), no longer assuming that closed equations for the populations exist.

\section{Quantum dynamical map}

The dynamical map $V(t)$ corresponding to the master equation (50) is defined as the solution of the integrodifferential equation

$$
\frac{d}{d t} V(t)=\int_{0}^{t} d \tau \mathcal{K}(\tau) V(t-\tau)
$$

with the initial condition $V(0)=I$, where $I$ denotes the identity map. Following Ref. [30] let us decompose the memory kernel as

$$
\mathcal{K}(\tau)=B(\tau)+C(\tau),
$$

where the superoperators $B(\tau)$ and $C(\tau)$ are defined by

$$
\begin{aligned}
B(\tau) \rho= & \sum_{\alpha} \gamma_{\alpha}(\tau) A_{\alpha}(\tau) \rho A_{\alpha}^{\dagger}(\tau), \\
C(\tau) \rho= & -i[H(\tau), \rho] \\
& -\frac{1}{2} \sum_{\alpha} \gamma_{\alpha}(\tau)\left\{A_{\alpha}^{\dagger}(\tau) A_{\alpha}(\tau), \rho\right\} .
\end{aligned}
$$

We further introduce the map $V_{0}(t)$ as the solution of the equation

$$
\frac{d}{d t} V_{0}(t)=\int_{0}^{t} d \tau C(\tau) V_{0}(t-\tau)
$$


with the initial condition $V_{0}(0)=I$. The Laplace transformation of Eqs. (54) and (58) yields

$$
\hat{V}(u)=\frac{1}{u-\hat{\mathcal{K}}(u)}, \quad \hat{V}_{0}(u)=\frac{1}{u-\hat{C}(u)},
$$

from which we get the Dyson-type identity

$$
\hat{V}(u)=\hat{V}_{0}(u)+\hat{V}_{0}(u) \hat{B}(u) \hat{V}(u) .
$$

Transforming back to the time domain we obtain the equation

$$
V(t)=V_{0}(t)+\left(V_{0} * B * V\right)(t) .
$$

Regarding formally the superoperator $B(\tau)$ as a perturbation and iterating Eq. (61) one finds that the full dynamical map $V(t)$ can be represented as a series,

$$
\begin{aligned}
V(t)= & V_{0}(t)+\left(V_{0} * B * V_{0}\right)(t) \\
& +\left(V_{0} * B * V_{0} * B * V_{0}\right)(t)+\ldots,
\end{aligned}
$$

which turns out to be a useful relation in the formulation of appropriate conditions for complete positivity.

\section{Sufficient conditions for complete positivity}

Let us first assume that the quantities $\gamma_{\alpha}(\tau)$ are positive functions, which means that the superoperator $B(\tau)$ defined by Eq. (56) is completely positive. Since the property of the complete positivity is preserved under addition and convolution, the representation (62) then tells us that the full dynamical map $V(t)$ is completely positive if the map $V_{0}(t)$ is completely positive. To bring this condition into an explicit form let us assume further that the Hermitian operators $H(\tau)$ and $\sum_{\alpha} \gamma_{\alpha}(\tau) A_{\alpha}^{\dagger}(\tau) A_{\alpha}(\tau)$ are diagonal in a time-independent orthonormal basis $\{|n\rangle\}$ for the underlying Hilbert space, i. e. we have

$$
\begin{aligned}
H(\tau) & =\sum_{n} \varepsilon_{n}(\tau)|n\rangle\langle n|, \\
\sum_{\alpha} \gamma_{\alpha}(\tau) A_{\alpha}^{\dagger}(\tau) A_{\alpha}(\tau) & =\sum_{n} k_{n}(\tau)|n\rangle\langle n|,
\end{aligned}
$$

with in general time-dependent eigenvalues $\varepsilon_{n}(\tau)$ and $k_{n}(\tau)$. Note that the positivity of the $\gamma_{\alpha}(\tau)$ implies that the eigenvalues $k_{n}(\tau)$ must be positive as well.

Equation (58) can now be solved to obtain

$$
V_{0}(t) \rho(0)=\sum_{n m} g_{n m}(t)|n\rangle\langle n|\rho(0)| m\rangle\langle m|,
$$

where the functions $g_{n m}(t)$ are the solutions of

$$
\dot{g}_{n m}(t)=-\int_{0}^{t} d \tau\left[z_{n}(\tau)+z_{m}^{*}(\tau)\right] g_{n m}(t-\tau),
$$

corresponding to the initial conditions $g_{n m}(0)=1$, and

$$
z_{n}(\tau)=\frac{1}{2} k_{n}(\tau)+i \varepsilon_{n}(\tau) .
$$

Equation (65) can be proven as follows. First, one shows that $C(\tau)(|n\rangle\langle m|)=-\left[z_{n}(\tau)+z_{m}^{*}(\tau)\right]|n\rangle\langle m|$. Using this relation and differentiating Eq. (58) one easily demonstrates that the expression (65) indeed represents the desired solution.

It is important to notice that the functions $g_{n n}(t)$ do actually coincide with the survival probabilities $g_{n}(t)$ introduced in Eq. (26). In fact, for $n=m$ we get from Eq. (66)

$$
\dot{g}_{n n}(t)=-\int_{0}^{t} d \tau k_{n}(\tau) g_{n n}(t-\tau) .
$$

The survival probabilities satisfy the same equation as can be seen by taking the time derivative of Eq. (26) and using Eq. (28).

One easily verifies that the representation (65) can be brought into the Kraus form (49) if and only if the matrix $G(t)$ with the elements $g_{n m}(t)$ is positive. Thus, we arrive at a sufficient condition for complete positivity: The quantum dynamical map $V(t)$ corresponding to the non-Markovian master equation (50) with the memory kernel (52) is completely positive if the condition

$$
G(t)=\left(g_{n m}(t)\right) \geq 0
$$

is fulfilled.

A necessary condition for (69) to hold is the positivity of the diagonal elements $g_{n n}(t)$ of $G(t)$ which coincide with the survival probabilities, $g_{n}(t)=g_{n n}(t)$. This necessary condition in turn implies the positivity of the functions $f_{n}(t)$ as can be seen immediately from Eq. (28) because $k_{n}(t) \geq 0$. Condition (69) therefore implies that the functions $f_{n}(t)$ allow an interpretation as true waiting time distributions. The positivity of the matrix $G(t)$ therefore represents a natural quantum generalization of the classical conditions for a semi-Markov process.

\section{Markovian limit and Lindblad theorem}

In the Markovian limit expressed by Eq. (51) we must have $k_{n}(\tau)=2 k_{n}^{0} \delta(\tau)$ and $\varepsilon_{n}(\tau)=2 \varepsilon_{n}^{0} \delta(\tau)$, such that $z_{n}(\tau)=2 z_{n}^{0} \delta(\tau)$. Equation (66) then reduces to the timelocal equation

$$
\dot{g}_{n m}(t)=-\left(z_{n}^{0}+z_{m}^{0 *}\right) g_{n m}(t),
$$

which is easily solved to yield

$$
g_{n m}(t)=h_{n}(t) h_{m}^{*}(t), \quad h_{n}(t)=e^{-z_{n}^{0} t} .
$$

Since a matrix with elements of this form is always positive we conclude that condition (69) is automatically satisfied. It follows from our results that the corresponding quantum dynamical map represents a completely positive semigroup. Hence, we see that our formulation correctly describes the transition to the Markovian limit and that it contains as a special case the "if"-part of the Lindblad theorem. 


\section{Negative memory functions}

Up to now we have considered the case that the superoperator $B(\tau)$ is completely positive which led to the sufficient condition (69) for the complete positivity of the dynamical map. The complete positivity of $B(\tau)$ implies that the memory functions must be positive, $k_{n}(t) \geq 0$. However, in many physical relevant applications these functions can take on negative values.

To include cases with negative kernel functions we consider the following general class of memory kernels. We use again the decomposition of the form given by Eq. (55), where $C(\tau)$ is given by the expressions (57), (63) and (64). However, we now drop the condition that the map $B(\tau)$ is completely positive, supposing instead that it takes the following general form,

$$
B(\tau)=\sum_{n} k_{n}(\tau) B_{n},
$$

where the $k_{n}(\tau)$ are real functions, not necessarily positive, and the $B_{n}$ are completely positive and timeindependent maps. The full memory kernel can thus be written as

$\mathcal{K}(\tau) \rho=-i[H(\tau), \rho]+\sum_{n} k_{n}(\tau)\left(B_{n} \rho-\frac{1}{2}\{|n\rangle\langle n|, \rho\}\right)$.

Without the assumption of the complete positivity of $B(\tau)$ the complete positivity of $V_{0}(t)$ is generally not sufficient for the complete positivity of the dynamical map $V(t)$. However, we can conclude from the representation (62) that $V(t)$ is completely positive if both $V_{0}(t)$ and $\left(V_{0} * B\right)(t)$ are completely positive. We have

$$
\left(V_{0} * B\right)(t) \rho=\sum_{l n m} f_{n m}^{l}(t)|n\rangle\left\langle n\left|B_{l} \rho\right| m\right\rangle\langle m|,
$$

where

$$
f_{n m}^{l}(t)=\int_{0}^{t} d \tau k_{l}(\tau) g_{n m}(t-\tau)
$$

The map (74) is completely positive if for all $l$ the matrix with elements $f_{n m}^{l}(t)$ is positive,

$$
F^{l}(t)=\left(f_{n m}^{l}(t)\right) \geq 0 .
$$

Summarizing, we have shown that the quantum dynamical map $V(t)$ is completely positive if the conditions (69) and (76) are fulfilled.

We can again provide the connection of the obtained results to the interpretation in terms a classical semiMarkov process. In fact, a necessary condition for (76) is the positivity of the functions $f_{n n}^{n}(t)$ which coincide with the functions $f_{n}(t)$. Conditions (69) and (176) thus imply that $g_{n}(t)$ and $f_{n}(t)$ can be interpreted as survival probability and as waiting time distribution for a classical semi-Markov process, respectively. These conditions therefore represent a generalization of the classical conditions to the quantum case for memory functions $k_{n}(t)$ that are allowed to take on negative values.

\section{Examples}

To illustrate the theory developed here and the various conditions for the complete positivity of the dynamical map we will now introduce a few examples. In particular we will consider a structure for the memory kernel which encompasses and generalizes a model recently studied in the literature for the description of memory effects [4, 21]. For this class of memory kernels we are in particular able to give necessary and sufficient conditions for the complete positivity of the dynamical evolution.

\section{Lattice systems}

Let us consider the following memory kernel,

$$
\begin{aligned}
\mathcal{K}(\tau) \rho= & -i[H(\tau), \rho]-\frac{1}{2} \sum_{n} k_{n}(\tau)\{|n\rangle\langle n|, \rho\}, \\
& +\sum_{m n} \pi_{m n} k_{n}(\tau)|m\rangle\langle n|\rho| n\rangle\langle m| .
\end{aligned}
$$

The special feature of this kernel is given by the fact that it leads to closed equations of motion for the populations $P_{n}(t)=\rho_{n n}(t)=\langle n|\rho(t)| n\rangle$ and for the coherences $\rho_{n m}(t)=\langle n|\rho(t)| m\rangle, n \neq m$. In fact, we find from the master equation with the above kernel that the coherences satisfy the same equation as the quantities $g_{n m}(t)$, namely Eq. (66). Taking into account the initial conditions we thus have

$$
\rho_{n m}(t)=g_{n m}(t) \rho_{n m}(0), \quad n \neq m .
$$

On the other hand, the populations are found to obey the generalized master equation (19) with $W_{n m}(\tau)=$ $\pi_{n m} k_{m}(\tau)$. We assume that this master equation describes a classical semi-Markov process and denote by $T_{n m}(t)$ the corresponding conditional transition probability. The diagonals of the density matrix can therefore be written as

$$
\rho_{n n}(t)=\sum_{m} T_{n m}(t) \rho_{m m}(0) .
$$

Thus, the memory kernel (77) may be viewed as describing a quantum particle moving on a lattice with sites labelled by $n$. The dynamics of the populations is modelled through a semi-Markov process with transition probabilities $\pi_{n m}$ and arbitrary waiting time distributions $f_{n}(t)$, while the $\rho_{n m}(t)$ describe the quantum coherences between different sites $n$ and $m$.

With the help of Eqs. (78) and (79) we can immediately construct the quantum dynamical map,

$$
\begin{aligned}
V(t) \rho(0)= & \sum_{n \neq m} g_{n m}(t)|n\rangle\langle n|\rho(0)| m\rangle\langle m| \\
& +\sum_{n m} T_{n m}(t)|n\rangle\langle m|\rho(0)| m\rangle\langle n| .
\end{aligned}
$$


Let us introduce a matrix $\tilde{G}(t)=\left(\tilde{g}_{n m}(t)\right)$ whose elements are defined by

$$
\tilde{g}_{n m}(t)=\left(T_{n n}(t)-g_{n n}(t)\right) \delta_{n m}+g_{n m}(t) .
$$

The off-diagonal elements of $\tilde{G}(t)$ thus coincide with those of the matrix $G(t)$ introduced earlier, while the diagonals of $\tilde{G}(t)$ are given by the conditional transition probabilities $T_{n n}(t)$. Then we can rewrite Eq. (80) as

$$
\begin{aligned}
V(t) \rho(0)= & \sum_{n m} \tilde{g}_{n m}(t)|n\rangle\langle n|\rho(0)| m\rangle\langle m| \\
& +\sum_{n \neq m} T_{n m}(t)|n\rangle\langle m|\rho(0)| m\rangle\langle n| .
\end{aligned}
$$

This is an exact formal representation for the full quantum dynamical map from which we infer that $V(t)$ is completely positive if and only if $\tilde{G}(t) \geq 0$ and $T_{n m}(t) \geq 0$ for all $n \neq m$. Being the conditional transition probabilities of a semi-Markov process, the $T_{n m}(t)$ always satisfy of course the second condition. Hence we obtain the result that the quantum dynamical map $V(t)$ is completely positive if and only if the condition

$$
\tilde{G}(t)=\left(\tilde{g}_{n m}(t)\right) \geq 0
$$

holds. This condition provides a full characterization of the complete positivity of the class of quantum semiMarkov processes given by Eq. (77).

Assuming the memory functions to be positive, the kernel (77) is easily seen to be of the form introduced in Sec. IIIC2 with the superoperator $B(\tau)$ given by the completely positive map

$$
B(\tau) \rho=\sum_{m n} \pi_{m n} k_{n}(\tau)|m\rangle\langle n|\rho| n\rangle\langle m| .
$$

Hence we can apply condition (69) as sufficient condition for the complete positivity of $V(t)$. We note that the probabilities $T_{n n}(t)$ are in general larger than the corresponding survival probabilities $g_{n}(t)=g_{n n}(t)$, since the process can be in the initial state $n$ at time $t$ both because it has not left it and because it has come back to it. According to Eq. (81) the necessary and sufficient condition (83) is therefore in general weaker than the merely sufficient condition (69). However, if the process involves only jumps in one specific direction, i. e., if the return probability vanishes for all states (this happens, e. g., for a purely decaying system), we have $T_{n n}(t)=g_{n}(t)$ and, hence, condition (69) becomes a necessary and sufficient condition for the complete positivity.

An instructive special case is that of a translational invariant system for which $k_{n}(\tau), g_{n m}(t)$ and $T_{n n}(t)$ are state-independent, i. e. $k_{n}(\tau)=k(\tau), g_{n m}(t)=g(t)$ and $T_{n n}(t)=T(t)$. The conditions (69) and (83) are then automatically fulfilled, showing that any such translational invariant process leads to a completely positive dynamical map. The same conclusion holds true if we allow the memory function $k(\tau)$ to become negative. In fact, the kernel (777) is also of the form used in Sec. IIIC4 with $B_{n}(\tau)$ given by

$$
B_{n} \rho=\sum_{m} \pi_{m n}|m\rangle\langle n|\rho| n\rangle\langle m| .
$$

Condition (76) is then also satisfied by assumption because it reduces to the condition that the waiting time distribution $f(\tau)$ corresponding to the memory function $k(\tau)$ must be positive.

\section{Exponential memory functions}

Memory kernels of the form (77) describe e.g. the dynamics of a two-level system interacting with a bosonic quantum reservoir, such as for example a two-level atom coupled to a damped field mode. The index $n$ now only takes on the two values + and - and the memory kernel reads

$$
\begin{aligned}
\mathcal{K}(\tau) \rho= & k_{+}(\tau)\left[\sigma_{-} \rho \sigma_{+}-\frac{1}{2}\left\{\sigma_{+} \sigma_{-}, \rho\right\}\right] \\
& +k_{-}(\tau)\left[\sigma_{+} \rho \sigma_{-}-\frac{1}{2}\left\{\sigma_{-} \sigma_{+}, \rho\right\}\right],
\end{aligned}
$$

where the jump probabilities are given by $\pi_{+-}=\pi_{-+}=$ 1 and $\pi_{--}=\pi_{++}=0$. A typical expression arising for the time dependence of the memory functions is given by [3, 4, 21]

$$
k_{ \pm}(\tau)=\kappa_{ \pm} e^{-\gamma \tau}
$$

with decay constants $\kappa_{ \pm} \geq 0$. This expression also allows an explicit evaluation of the relevant quantities such as the functions $g_{n m}(t)$ and the conditional transition probabilities $T_{n m}(t)$. Inverting Eq. (31) to obtain

$$
\hat{f}_{ \pm}(u)=\frac{\hat{k}_{ \pm}(u)}{u+\hat{k}_{ \pm}(u)},
$$

and further calculating the inverse Laplace transform one finds the functions

$$
f_{ \pm}(\tau)=2 \frac{\kappa_{ \pm}}{d_{ \pm}} e^{-\gamma \tau / 2} \sinh \left(d_{ \pm} \tau / 2\right)
$$

with

$$
d_{ \pm}=\sqrt{\gamma^{2}-4 \kappa_{ \pm}}
$$

The functions $f_{ \pm}(\tau)$ are positive and normalized to one (or identically zero) if and only if

$$
\frac{\gamma^{2}}{4} \geq \max \left\{\kappa_{+}, \kappa_{-}\right\}
$$

so that only in this case they can be interpreted as waiting time distributions and the generalized master equation corresponding to Eq. (86) describes a classical semiMarkov process. In fact under the condition $\gamma^{2} \geq 4 \kappa_{+}$ 
the function $f_{+}(\tau)$ can be expressed as a difference of two exponentials as in (39) with the identifications

$$
\lambda_{1,2}=\frac{\gamma}{2} \pm \frac{1}{2} \sqrt{\gamma^{2}-4 \kappa_{+}},
$$

thus corresponding to a generalized Erlang distribution, and similarly for the function $f_{-}(\tau)$.

The conditional transition probabilities for the associated semi-Markov process can be calculated observing that due to Eq. (79) the quantity $T_{++}(t)$ is given by the solution $\rho_{++}(t)$ of the master equation obtained with the initial condition $\rho_{++}(0)=1$ and similarly for $T_{--}(t)$. Starting from Eq. (86) one has the equation

$$
\dot{\rho}_{++}(t)=\int_{0}^{t}\left[k_{-}(t-\tau) \rho_{--}(\tau)-k_{+}(t-\tau) \rho_{++}(\tau)\right]
$$

Differentiating this equation with respect to time and exploiting the exponential form of the memory functions (87) together with the trace preservation one is led to a telegraph equation of the form

$$
\ddot{\rho}_{++}(t)+\gamma \dot{\rho}_{++}(t)+\left(\kappa_{+}+\kappa_{-}\right) \rho_{++}(t)-\kappa_{-}=0 .
$$

Its solution with the initial conditions $\rho_{++}(0)=1$ and $\dot{\rho}_{++}(0)=0$ gives the conditional transition probability $T_{++}(t)$, and an analogous calculation can be performed for $T_{--}(t)$. The results read

$$
\begin{aligned}
T_{++}(t)= & \frac{\kappa_{-}}{\kappa_{+}+\kappa_{-}} \\
& +\frac{\kappa_{+}}{\kappa_{+}+\kappa_{-}} e^{-\gamma t / 2}\left[\cosh (d t / 2)+\frac{\gamma}{d} \sinh (d t / 2)\right], \\
T_{--}(t)= & \frac{\kappa_{+}}{\kappa_{+}+\kappa_{-}} \\
& +\frac{\kappa_{-}}{\kappa_{+}+\kappa_{-}} e^{-\gamma t / 2}\left[\cosh (d t / 2)+\frac{\gamma}{d} \sinh (d t / 2)\right]
\end{aligned}
$$

with

$$
d=\sqrt{\gamma^{2}-4\left(\kappa_{+}+\kappa_{-}\right)}
$$

Note that despite the fact that $d$ is not necessarily real, since this is generally not implied by Eq. (91), the transition probabilities themselves are always positive.

For the memory functions (87) one can also exactly calculate the entries of the matrix $G(t)$ considered in (69). In fact both $g_{++}(t)$ and $g_{--}(t)$ due to (68) and the exponential form of the kernel have a second derivative which obeys a telegraph equation, and similarly for $g_{+-}(t)$. The corresponding solutions read

$$
\begin{aligned}
& g_{++}(t)=e^{-\gamma t / 2}\left[\cosh \left(d_{+} t / 2\right)+\frac{\gamma}{d_{+}} \sinh \left(d_{+} t / 2\right)\right] \\
& g_{--}(t)=e^{-\gamma t / 2}\left[\cosh \left(d_{-} t / 2\right)+\frac{\gamma}{d_{-}} \sinh \left(d_{-} t / 2\right)\right]
\end{aligned}
$$

and

$$
g_{+-}(t)=e^{-\gamma t / 2}\left[\cosh (\bar{d} t / 2)+\frac{\gamma}{\bar{d}} \sinh (\bar{d} t / 2)\right],
$$

with $d_{ \pm}$as in Eq. (90) while

$$
\bar{d}=\sqrt{\gamma^{2}-2\left(\kappa_{+}+\kappa_{-}\right)} .
$$

In particular $g_{+-}(t)=g_{-+}(t)$ since it is a real quantity. Note that indeed minus the derivative of $g_{++}(t)$ is equal to $f_{+}(t)$, according to its interpretation as survival probability, and similarly for $g_{--}(t)$ and $f_{-}(t)$. This entails in particular that both $g_{++}(t)$ and $g_{--}(t)$ are positive non-increasing functions taking the value one for $t=0$.

One can check that as discussed in Sec. IIID 1 indeed the inequality $T_{n n}(t) \geq g_{n n}(t)$ generally holds with $n=$ \pm . In fact the quantity $T_{n n}(t)-g_{n n}(t)$ is equal to zero for $t=0$ and has a non-negative derivative with respect to time for all values of the parameters complying with Eq. (91). This is consistent with the interpretation of the $T_{n n}(t)$ as conditional transition probabilities which must be larger or equal to the corresponding survival probabilities. In particular one immediately sees from the explicit expressions (95) and (97) that for $\kappa_{-}=0$, so that one only has transitions in one direction, $T_{++}(t)=$ $g_{++}(t)$ and $T_{--}(t)=g_{--}(t)=1$.

The necessary and sufficient conditions for complete positivity of the dynamics described by Eq. (86) according to the general result Eq. (83) are thus given by

$$
T_{++}(t) T_{--}(t) \geq g_{-+}^{2}(t),
$$

together with the constraint (91), which ensures that the populations obey a generalized master equation describing a classical semi-Markov process. One therefore has to find out the possible range of parameters $\kappa_{+}, \kappa_{-}$and $\gamma$ for which the inequality Eq. (100) is satisfied for all times, or at least up to a certain time. The task of finding conditions for complete positivity of the dynamics given by Eq. (86) is indeed far from trivial and has been accomplished only partially in the literature [4, 21].

Taking the explicit expressions (95) and (97) into account and noting that for $t=0 \mathrm{Eq}$. (100) is actually an equality, one can look at the short-time behavior of the quantity

$$
\Delta(t)=T_{++}(t) T_{--}(t)-g_{-+}^{2}(t) .
$$

In view of the constraint Eq. (91) the function $\Delta$ is most conveniently expressed in terms of the rescaled quantities

$$
r_{ \pm}=\frac{4}{\gamma^{2}} \kappa_{ \pm}
$$

bound to the interval $[0,1]$, and $\tau=\gamma t$. In terms of these new variables $\Delta$ can be written as

$$
\begin{aligned}
\Delta(\tau)= & \left(\frac{r_{-}}{r_{-}+r_{+}}+\frac{r_{+}}{r_{-}+r_{+}} e^{-\tau / 2} h_{1}(\tau)\right) \\
& \times\left(\frac{r_{+}}{r_{-}+r_{+}}+\frac{r_{-}}{r_{-}+r_{+}} e^{-\tau / 2} h_{1}(\tau)\right) \\
& -e^{-\tau} h_{2}^{2}(\tau),
\end{aligned}
$$




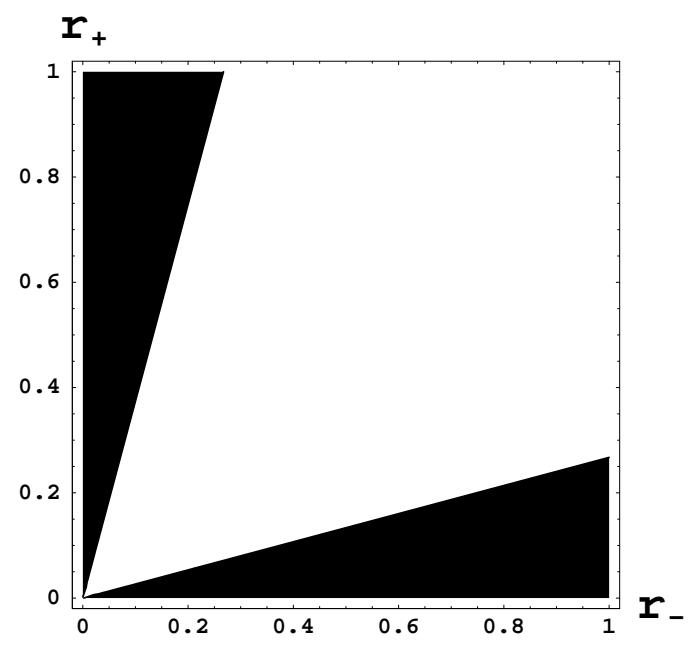

FIG. 1: The sign of $\Delta(\tau)$ plotted for $\tau=0.01$. Within the white region $\Delta$ is positive, while it is negative in the black regions. The range of $r_{ \pm}$is restricted to $[0,1]$ according to the constraint (91).

where

$$
\begin{array}{r}
h_{1}(\tau)=\cosh \left(\frac{\tau}{2} \sqrt{1-\left(r_{-}+r_{+}\right)}\right) \\
+\frac{\sinh \left(\frac{\tau}{2} \sqrt{1-\left(r_{-}+r_{+}\right)}\right)}{\sqrt{1-\left(r_{-}+r_{+}\right)}}
\end{array}
$$

and

$$
\begin{aligned}
h_{2}(\tau)= & \cosh \left(\frac{\tau}{2} \sqrt{1-\left(r_{-}+r_{+}\right) / 2}\right) \\
+ & \frac{\sinh \left(\frac{\tau}{2} \sqrt{1-\left(r_{-}+r_{+}\right) / 2}\right)}{\sqrt{1-\left(r_{-}+r_{+}\right) / 2}} .
\end{aligned}
$$

The Taylor expansion of $\Delta$ for small $\tau$ reads

$$
\Delta(\tau)=-\frac{1}{96}\left(r_{+}^{2}+r_{-}^{2}-4 r_{+} r_{-}\right) \tau^{3}+\mathcal{O}\left(\tau^{4}\right)
$$

so that whenever

$$
r_{+}^{2}+r_{-}^{2}-4 r_{+} r_{-}>0
$$

one has violation of complete positivity for very short times. This is obviously the case for either $r_{-}=0$, $r_{+}>0$ or $r_{+}=0, r_{-}>0$. The function $\Delta$ is plotted in Fig. 1]in the region allowed by Eq. (91), still clearly taking on negative values. Note that in standard physical situations one has $r_{+} \geq r_{-}$, which corresponds to positivity of the reservoir temperature. Indeed the condition $r_{-}=0$ would correspond to a zero temperature reservoir. This remark confirms a result obtained in a completely different way in 21].

For $r_{-}>0$ the inequality (107) can be rewritten as

$$
\left(\frac{r_{+}}{r_{-}}\right)^{2}-4\left(\frac{r_{+}}{r_{-}}\right)+1>0,
$$

which is satisfied whenever

$$
r_{+}<(2-\sqrt{3}) r_{-} \quad \text { or } \quad r_{+}>(2+\sqrt{3}) r_{-} .
$$

In these parameter regions, even when the classical condition Eq. (91) holds, the necessary and sufficient condition Eq. (100) for complete positivity is violated for very short times. Assuming as discussed above $r_{+} \geq r_{-}$we have thus obtained that the decay constants must satisfy the constraint

$$
r_{-} \leq r_{+} \leq(2+\sqrt{3}) r_{-}
$$

so as to avoid loss of complete positivity for very short times. A numerical analysis indicates that in this parameter region the inequality Eq. (100) is satisfied, corresponding to preservation of complete positivity. Indeed the triangular white region in Fig. 1, which corresponds to positivity of the quantity $\Delta$ and therefore to fulfilment of the condition (100), gets larger with growing time. In Fig. 2 the quantity $\Delta$ is plotted as a function of $\tau$ and of the ratio $r_{+} / r_{-}$. For the case of the spin-boson model considered in [21], where the decay constants $\kappa_{ \pm}$are expressed in terms of the mean number of excitations of the reservoir at the frequency $\omega$ of the two-level system, taking Eq. (102) into account the constraint Eq. (110) is equivalent to

$$
\beta \hbar \omega \leq \ln (2+\sqrt{3}) .
$$

The time evolution is therefore completely positive only for reservoir temperatures above a certain threshold given by $k_{B} T \approx 0.8 \hbar \omega$.

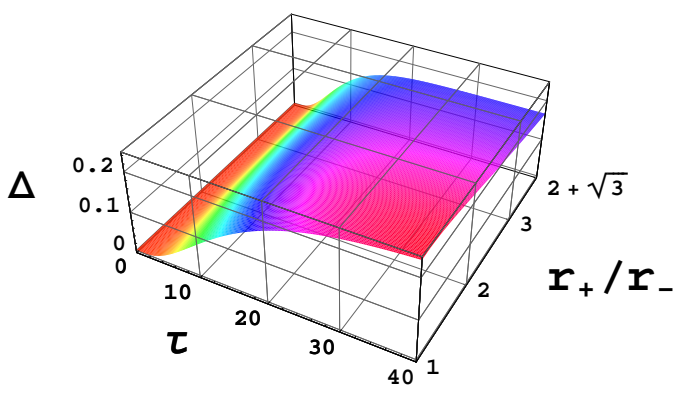

FIG. 2: (Color online) The quantity $\Delta$ plotted as a function of $\tau$ and of the ratio between the decay constants in the range $[1,2+\sqrt{3}] . r_{-}$is fixed to be 0.2 .

In particular for $r_{+}=r_{-}$, which corresponds to an infinite temperature reservoir, complete positivity is granted on the basis of the classical condition Eq. (91) only, as already discussed in Sec. IIID 1, and confirmed by other approaches [4, 21]. In fact in this case $T_{++}(t)=$ $T_{--}(t)=T(t)$ and $g_{++}(t)=g_{--}(t)=g(t)$, so that 
the condition (100) reduces to $T(t) \geq g(t)$, which in our approach is automatically known to be true because of the probabilistic interpretation of the quantities involved, and corresponds to the inequality (22) in Ref. [21].

\section{CONCLUSIONS}

We have constructed a quantum mechanical generalization of the classical concept of semi-Markov processes and discussed the basic features of the resulting quantum semi-Markov processes, including, in particular, the formulation of mathematical criteria for the complete positivity of the corresponding quantum dynamical maps. The main motivation of our study was the development of a structural characterization of non-Markovian dynamics for a large class of quantum processes that is relevant for physical applications. The approach followed here could indeed be particularly useful in applications for which a microscopic system-environment approach is technically too complicated or impossible, guiding the phenomenological construction of the memory kernel.

It it important to stress that the class of quantum semiMarkov processes considered here do contain the Markovian limit as a straightforward special case as shown at the end of Sec. ПIIC thus providing a natural generalization of quantum Markov processes. In our derivation of the various conditions for complete positivity we have made some specific assumptions in order to obtain explicit constraints. For example, we have assumed a certain structure for the Hamiltonian part and for the loss term of the memory kernel. More general quantum semi-Markov processes can be considered, and will be the object of future investigations. In particular, we notice that the conditions obtained in Sec. IIIC, which are only sufficient, could be too stringent in certain physical applications. It is therefore important to study further examples in order to decide whether or not a generalization of these criteria is necessary in practice. This is particularly true for the case of temporarily negative memory functions (see Sec. III C 4). However, as is shown in the examples of Sec. IIID, for specific cases one can formulate conditions for the complete positivity which are not only sufficient but also necessary, thus leading to a complete characterization of physically admissible memory kernels. This has been done for the memory kernel of Eq. (86) which describes a two-level system interacting with a bosonic reservoir, extending the partial analysis given in [4, 21]. It has been shown that Eq. (110), together with the constraint (91) for the allowed region of decay constants, is indeed a necessary condition for complete positivity, and numerical evidence strongly suggests that this condition is also sufficient. This criterion for complete positivity can also be understood as a bound on the temperature range over which the model can give physically well-defined results.

\section{Acknowledgments}

One of us (HPB) gratefully acknowledges financial support from the Hanse-Wissenschaftskolleg, Delmenhorst.
[1] V. Gorini, A. Kossakowski, and E. C. G. Sudarshan, J. Math. Phys. 17, 821 (1976)

[2] G. Lindblad, Comm. Math. Phys. 48, 119 (1976)

[3] H.-P. Breuer and F. Petruccione, The Theory of Open Quantum Systems (Oxford University Press, Oxford, 2007)

[4] A. A. Budini, Phys. Rev. A 69, 042107 (2004)

[5] A. A. Budini, Phys. Rev. E 72, 056106 (2005)

[6] A. A. Budini and H. Schomerus, J. Phys. A: Math. Gen. 38, 9251 (2005)

[7] A. A. Budini, Phys. Rev. A 74, 053815 (2006)

[8] H.-P. Breuer, J. Gemmer, and M. Michel, Phys. Rev. E 73, 016139 (2006)

[9] H.-P. Breuer, Phys. Rev. A 75, 022103 (2007)

[10] B. Vacchini, Phys. Rev. A 78, 022112 (2008)

[11] J. Piilo, S. Maniscalco, K. Harkonen, and K.-A. Suominen, Phys. Rev. Lett. 100, 180402 (2008)

[12] E. Ferraro, H.-P. Breuer, A. Napoli, M. A. Jivulescu, and A. Messina, Phys. Rev. B 78, 064309 (2008)

[13] H. Krovi, O. Oreshkov, M. Ryazanov, and D. A. Lidar, Phys. Rev. A 76, 052117 (2007)

[14] W. Feller, Proc. Nat. Acad. Sci. U.S.A. 51, 653 (1964)

[15] W. Feller, An introduction to probability theory and its applications. Vol. II (John Wiley \& Sons, New York, 1971)
[16] D. R. Cox and H. D. Miller, The theory of stochastic processes (John Wiley \& Sons, New York, 1965)

[17] V. Nollau, Semi-Markovsche Prozesse (Akademie-Verlag, Berlin, 1980)

[18] B. D. Hughes, Random walks and random environments. Vol. 1 (Clarendon Press, Oxford, 1995)

[19] H.-P. Breuer and B. Vacchini, Phys. Rev. Lett. 101, 140402 (2008)

[20] S. M. Barnett and S. Stenholm, Phys. Rev. A 64, 033808 (2001)

[21] S. Maniscalco, Phys. Rev. A 75, 062103 (2007)

[22] J. Wilkie and Y. M. Wong, J. Phys. A: Math. Gen. 42, 015006 (2009)

[23] S. M. Ross, Introduction to probability models (Academic Press, Burlington, MA, 2007)

[24] D. T. Gillespie, Phys. Lett. A 64, 22 (1977)

[25] P. Allegrini, G. Aquino, P. Grigolini, L. Palatella, and A. Rosa, Phys. Rev. E 68, 056123 (2003)

[26] S. Nakajima, Progr. Theor. Phys. 20, 948 (1958)

[27] R. Zwanzig, J. Chem. Phys. 33, 1338 (1960)

[28] S. Daffer, K. Wódkiewicz, J. D. Cresser, and J. K. McIver, Phys. Rev. A 70, 010304 (R) (2004)

[29] A. Shabani and D. A. Lidar, Phys. Rev. A 71, 020101 (R) (2005)

[30] A. Kossakowski and R. Rebolledo, Open Syst. Inf. Dyn. 
15, 135 (2008) 